\title{
On the forward cone quantization of the Dirac field in "longitudinal boost-invariant" coordinates with cylindrical symmetry
}

\author{
Bogdan Mihaila, , 田 John F. Dawson, 2 , 团 and Fred Cooper ${ }^{3,4}$, 团 \\ ${ }^{1}$ Materials Science and Technology Division, Los Alamos National Laboratory, Los Alamos, NM 87545 \\ ${ }^{2}$ Department of Physics, University of New Hampshire, Durham, NH 03824 \\ ${ }^{3}$ Santa Fe Institute, Santa Fe, NM 87501 \\ ${ }^{4}$ Theoretical Division, Los Alamos National Laboratory, Los Alamos, NM 87545
}

\begin{abstract}
We obtain a complete set of free-field solutions of the Dirac equation in a (longitudinal) boostinvariant geometry with azimuthal symmetry and use these solutions to perform the canonical quantization of a free Dirac field of mass $M$. This coordinate system which uses the $1+1$ dimensional fluid rapidity $\eta=1 / 2 \ln [(t-z) /(t+z)]$ and the fluid proper time $\tau=\left(t^{2}-z^{2}\right)^{1 / 2}$ is relevant for understanding particle production of quarks and antiquarks following an ultrarelativistic collision of heavy ions, as it incorporates the (approximate) longitudinal "boost invariance" of the distribution of outgoing particles. We compare two approaches to solving the Dirac equation in curvilinear coordinates, one directly using Vierbeins, and one using a "diagonal" Vierbein representation.
\end{abstract}

PACS numbers: 25.75.-q, 04.60.Ds

\section{INTRODUCTION}

The kinematics of heavy-ion collisions at relativistic colliders is such that the distribution of secondary particles obeys an approximate longitudinal boost invariance. This approximate invariance was the basis for many hydrodynamical simulations of particle production following the collision of two ultrarelativistic heavy ions. The use of this symmetry for heavy-ion collisions was advocated by Bjorken [1] in a seminal paper which was based on earlier work using Landau's hydrodynamic model [2] by Cooper, Frye and Schonberg 3] and Hwa 4]. In a hydrodynamic description of the time evolution of the plasma produced following a collision, this invariance leads to the surfaces of constant energy density being functions primarily of the kinematic variable $\tau=\left(t^{2}-z^{2}\right)^{1 / 2}$, where the $z$ axis is the axis of cylindrical symmetry. For head-on collisions of identical nuclei, the kinematics also has cylindrical symmetry.

In trying to understand the particle production of hadrons in this scenario from first principles, one approach is to assume that a significant fraction of the initial energy that is eventually transformed into the final hadrons is in a classical gluonic field configuration which produces quarks and quantum gluons by the Schwinger tunneling mechanism [5]. In this approximation the particles are produced by nonperturbative tunneling. However, we ignore production due to further quantum corrections due to rescattering of the quarks and gluons that are "sparked" from the vacuum.

In this boost-invariant situation, all physical quantities such as energy densities are just functions of the proper time $\tau$. In particular the chromoelectric field density $\vec{E}_{\alpha}$,

\footnotetext{
*Electronic address: bmihaila@lanl.gov

†Electronic address: john.dawson@unh.edu

$\ddagger$ Electronic address: cooper@santafe.edu
}

$\alpha=1,2, \ldots 8$ is also a function of $\tau$. Choosing the chromoelectric field in the longitudinal direction, then we can choose a gauge where the vector potential is also a function only of $u=\ln \tau$ and is defined via the relationship

$$
E_{\alpha}^{3}(u)=-\frac{\partial A_{\alpha}^{3}(u)}{\partial u}
$$

In this picture the "classical gluonic field" $A_{\alpha}^{3}$ would approximately be a function of $\tau$ and one would obtain the initial quantum plasma by studying the coupled problem of the produced quarks and gluons interacting with the background classical gluonic field. Such a picture was advocated by Cooper et al. [6] for the production of particles in the central rapidity region. Once such a system of equations is solved, one then has to construct the physical quantities of QCD which depend on the two Casimir invariants of color $\mathrm{SU}(3)$. It is only these quantities which are gauge invariant.

In the hydrodynamic scaling regime where the fluid velocity $v=z / t$, the fluid rapidity $\eta=1 / 2 \ln [(t-z) /(t+z)]$ can be identified with the particle rapidity: $1 / 2 \ln [(1-$ $v) /(1+v)$ ]. In Ref. [6] numerical simulations were performed in $1+1$ dimensions, with an a Abelian external field. Although the formalism for doing the $3+1$ dimensional calculation was presented, simulations were not done in that paper and the cylindrical symmetry of the head-on collision problem was not exploited. By exploiting the cylindrical symmetry, the computational complexity of the back reaction problem is reduced from $N^{3}$ modes to describe the quantum field of the quarks to $N^{2}$ modes which is quite a savings.

Recently the problem of finding the transverse momentum distribution of quarks and gluons produces by a strong constant chromoelectric field by the Schwinger mechanism [5] has been solved [7]. This calculation found the interesting physical result that the transverse distributions of produced particles depends not only on an invariant related to the energy density of the plasma, but also an invariant which describes the direction in color 
space that the initial chromoelectric field was pointing. This might have important experimental consequences. In this paper we will discuss the canonical quantization of the free Dirac field in the coordinate system which utilizes the curvilinear coordinates $\tau$ and the "fluid" longitudinal rapidity $\eta=\frac{1}{2} \ln [(t-z) /(t+z)]$ as well as the cylindrical coordinates $\rho$ and $\phi$ that correspond to the directions transverse to the collision axis. This formulation will simplify identifying the adiabatic solutions to the back reaction problem and will simplify the computational effort needed to determine transverse momentum distributions. The canonical quantization of the fermion field presented here will also allow us to precisely define the initial state of the quarks before the chromoelectric field is "turned on", so that the initial values of the time evolution problem and back reaction can be specified.

The problem of quantizing the Dirac field in an arbitrary coordinate system was first discussed in the classic paper of Brill and Wheeler [8] who were concerned with understanding the motion of electrons and neutrinos in a background gravitational field. This problem was also later considered by Parker [9] and collaborators who were interested in the problem of particle creation in expanding universes. More recently, Viallalba and coworkers have concerned themselves with the general problem of separation of variables [10] in external vector fields in order to find exact solutions [11] of the Dirac equation. The problem of quantizing the Dirac equation on the hyperboloids $\tau=\left(t^{2}-x^{2}-y^{2}-z^{2}\right)^{1 / 2}$ was also studied in great detail in the 1970's by Sommerfield [12], diSessa [13] and others [14]. Furthermore, the problem of quantizing the Dirac equation in cylindrical coordinates was studied by Balantekin and DeWeerd [15] also with interest in ultrarelativistic heavy-ion collisions, but without exploiting the boost invariance aspect of this problem.

The intent of this paper is to fill the apparent gap in the previous work on this subject by studying the Dirac equation in a situation where the $1+1$ dimensional fluid proper time $\tau=\left(t^{2}-z^{2}\right)^{1 / 2}$ is important as well as the cylindrical symmetry. In doing so we will also compare and relate two different ways of considering this problem, first directly using Vierbeins (tetrads) [16] to transform into the curvilinear coordinate system, and the second making a similarity transform into a frame where the Vierbeins are diagonal.

The paper is organized as follows: In Sec. II we will review the general mathematical framework of the subsequent discussion and outline our approach. In Sec. III we will discuss the $1+1$ realization of this problem using boost-invariant coordinates. In Sec. IV we will discuss the case of a $3+1$ dimensional Dirac equation in cylindrical coordinates. Finally, in Sec. V w we will combine the results of the previous two sections in order to discuss the solution of the Dirac equation in longitudinal boostinvariant coordinates with azimuthal symmetry. We conclude in Sec. VI.

\section{GENERAL FRAMEWORK}

The free-field Dirac equation in Cartesian coordinates $\left(\xi^{\alpha}\right)$ is given by

$$
\left(\gamma^{\alpha} \partial_{\alpha}+M\right) \psi=0
$$

Throughout this paper we use the metric

$$
\eta^{\alpha \beta}=\operatorname{diag}(-1,1,1,1)
$$

and the Dirac gamma matrices in the chiral representation, in 3+1 dimensions, given as

$$
\gamma^{0}=\left[\begin{array}{cc}
0 & 1 \\
-1 & 0
\end{array}\right], \quad \gamma^{k}=\left[\begin{array}{cc}
0 & \sigma_{k} \\
\sigma_{k} & 0
\end{array}\right],
$$

where $\sigma^{k}$ are the usual Pauli matrices, and 1 is the unit $(2 \times 2)$ matrix. The constant Dirac matrices satisfy the relation

$$
\left\{\gamma^{\alpha}, \gamma^{\beta}\right\}=2 \eta^{\alpha \beta}
$$

Next, we will consider the change of variables $\left(\xi^{\alpha} \rightarrow x^{a}\right)$. We introduce the Vierbeins, $\mathrm{V}_{a}^{\alpha}$, and their inverses, $\mathbf{V}^{a}{ }_{\alpha}$, as

$$
\begin{array}{r}
\mathrm{d} \xi^{\alpha}=\frac{\partial \xi^{\alpha}}{\partial x^{a}} \mathrm{~d} x^{a}=\mathrm{V}^{\alpha}{ }_{a} \mathrm{~d} x^{a} \\
\partial_{\alpha}=\frac{\partial}{\partial \xi^{\alpha}}=\frac{\partial x^{a}}{\partial \xi^{\alpha}} \frac{\partial}{\partial x^{a}}=\mathbf{V}^{a}{ }_{\alpha} \partial_{a} .
\end{array}
$$

We have

$$
\mathrm{V}_{a}^{\alpha} \mathbf{V}_{\beta}^{a}=\delta_{\alpha \beta}, \quad \mathbf{V}_{\alpha}^{a} \mathrm{~V}_{b}^{\alpha}=\delta_{a b} .
$$

Under the change of variables the line element transforms as

$$
\mathrm{d} s^{2}=\eta_{\alpha \beta} \mathrm{d} \xi^{\alpha} \mathrm{d} \xi^{\beta}=g_{a b} \mathrm{~d} x^{a} \mathrm{~d} x^{b} .
$$

In the following we will use the notation $\sqrt{-g}=\operatorname{det}\left[g_{a b}\right]$. One also has the connection between the Cartesian metric and the metric in the curvilinear coordinates

$$
g_{a b}=\mathbf{V}_{a}^{\alpha} \mathbf{V}_{b}^{\beta} \eta_{\alpha \beta}
$$

Finally, in the new system of coordinates the free-field Dirac equation (2.1) becomes

$$
\left(\tilde{\gamma}^{a} \partial_{a}+M\right) \psi=0
$$

To simplify the Dirac equation in an arbitrary curvilinear coordinate system one often performs a similarity transformation to diagonalize the Vierbein matrices. This then produces a representation which we will connote the "diagonal tetrad" representation. To obtain the diagonal tetrad representation, one introduces a similarity transformation, $S$, such that

$$
S^{-1} \tilde{\gamma}^{a} S=\bar{\gamma}^{a}
$$


where the gamma matrices $\bar{\gamma}^{a}$ are proportional to the constant gamma matrices, $\gamma^{a}$. By construction, the two set of gamma matrices, $\tilde{\gamma}^{a}$ and $\bar{\gamma}^{a}$, are defined such that

$$
\bar{\gamma}^{\alpha}=\overline{\mathbf{V}}_{\alpha}^{a} \gamma^{\alpha}
$$

where $\overline{\mathbf{V}}_{\alpha}^{a}$ are the Vierbeins in the diagonal tetrad representation. The two sets of gamma matrices, $\tilde{\gamma}^{a}$ and $\bar{\gamma}^{a}$ satisfy the same algebra

$$
\left\{\tilde{\gamma}^{a}, \tilde{\gamma}^{b}\right\}=\left\{\bar{\gamma}^{a}, \bar{\gamma}^{b}\right\}=2 g^{a b}
$$

Hence, the similarity transformation $S$ which connects these two irreducible sets of matrices, is unique [17].

In the diagonal tetrad representation the Dirac equation becomes

$$
\left(\bar{\gamma}^{a} \nabla_{a}+M\right) \psi_{[\mathrm{d}]}=0
$$

where we have introduced the diagonal Dirac wave function

$$
\psi_{[\mathrm{d}]}=S^{-1} \psi,
$$

and the covariant derivatives

$$
\nabla_{a}=S^{-1} \partial_{a} S=\partial_{a}-\Gamma_{a}
$$

Here, the spinor connection $\Gamma_{a}$ is given by

$$
\Gamma_{a}=\frac{1}{2} \Sigma^{\alpha \beta} \eta_{\alpha \gamma} \overline{\mathbf{V}}_{b}^{\gamma}\left(\partial_{a} \overline{\mathbf{V}}_{\beta}^{b}+\Gamma_{a c}^{b} \overline{\mathbf{V}}_{\beta}^{c}\right)
$$

and we have introduced the notation $\Sigma^{\alpha \beta}=\frac{1}{4}\left[\gamma^{\alpha}, \gamma^{\beta}\right]$, defined in terms of the constant Dirac matrices.

The Christoffel symbols, $\Gamma_{b c}^{a}$, are obtained from the Lagrange equations of motion

$$
\frac{\mathrm{d}}{\mathrm{d} t}\left(\frac{\partial \mathcal{L}}{\partial \dot{x}^{a}}\right)-\frac{\partial \mathcal{L}}{\partial x^{a}}=0
$$

or

$$
\ddot{x}^{a}+\Gamma_{b c}^{a} \dot{x}^{b} \dot{x}^{c}=0
$$

corresponding to the Lagrangian of a free particle in the $x^{a}$ coordinates [18], i.e.

$$
\mathcal{L}=g_{a b} \dot{x}^{a} \dot{x}^{b} .
$$

The inner product is defined by considering the covariant conservation law for a current. In an arbitrary number $(d)$ of spatial dimensions, we have the conservation law [19]

$$
\int \mathrm{d}^{d+1} x \sqrt{-g} \mathcal{V}_{; \mu}^{\mu}=0
$$

provided that $\mathcal{V}^{\mu}$ vanishes at infinity. Here the covariant divergence is defined as

$$
\mathcal{V}_{; \mu}^{\mu}=\frac{1}{\sqrt{-g}} \partial_{\mu}\left(\sqrt{-g} \mathcal{V}^{\mu}\right)
$$

and the conservation law can also be written as

$$
\int \mathrm{d}^{d+1} x \partial_{\mu}\left(\sqrt{-g} \mathcal{V}^{\mu}\right)=0 \text {. }
$$

or

$$
\int \mathrm{d} \Sigma^{\mu} \sqrt{-g} \mathcal{V}^{\mu}=0 .
$$

Here $\Sigma^{\mu}$ is the $d$-dimensional hyper-surface perpendicular to the $\mu$ direction. Choosing $\mu=0$ then the conserved charge is (with $\mathcal{V}^{\mu} \equiv j^{\mu}=\bar{\psi} \tilde{\gamma}^{\mu} \psi$ )

$$
Q=\int_{x_{0}=\text { const. }} \mathrm{d}^{d} x \sqrt{-g}\left(\bar{\psi} \tilde{\gamma}^{0} \psi\right) .
$$

Thus the natural Dirac inner product is :

$$
\left(\psi_{1}, \psi_{2}\right) \equiv \int_{x_{0}=\text { const. }} \mathrm{d}^{d} x \quad \sqrt{-g}\left(\bar{\psi}_{1} \tilde{\gamma}^{0} \psi_{2}\right),
$$

with $\bar{\psi} \equiv \psi^{\dagger} \gamma^{0}$. The canonical quantization of the Dirac field assumes a mode expansion:

$$
\hat{\psi}(x)=\int \mathrm{d}^{d} k\left[\psi_{\mathbf{k}}^{(+)}(x) a_{\mathbf{k}}+\psi_{\mathbf{k}}^{(-)}(x) b_{\mathbf{k}}^{\dagger}\right] .
$$

with

$$
\left\{a_{\mathbf{k}}, a_{\mathbf{k}^{\prime}}^{\dagger}\right\}=\left\{b_{\mathbf{k}}, b_{\mathbf{k}^{\prime}}^{\dagger}\right\}=\delta\left(\mathbf{k}^{\prime}-\mathbf{k}\right) .
$$

Here $\psi_{k}^{( \pm)}(x)$ are positive- and negative-energy solutions [20] of the homogeneous Dirac equation and satisfy the orthonormality relations

$$
\begin{aligned}
& \left(\bar{\psi}_{\mathbf{k}}^{( \pm)}, \psi_{\mathbf{k}^{\prime}}^{( \pm)}\right)=\delta\left(\mathbf{k}^{\prime}-\mathbf{k}\right) \\
& \left(\bar{\psi}_{\mathbf{k}}^{(+)}, \psi_{\mathbf{k}^{\prime}}^{(-)}\right)=0 .
\end{aligned}
$$

In the following, we will study the canonical quantization of the free Dirac field in the Cartesian and diagonal tetrad representations, in the context of several system of coordinates characteristic to the physics of heavy-ion collisions.

\section{BOOST-INVARIANT COORDINATES IN 1+1 DIMENSIONS}

For illustrative purposes, we consider first the $1+1$ dimensional case studied previously by diSessa [13]. Here, the Dirac equation is

$$
\left(\gamma^{0} \partial_{0}+\gamma^{3} \partial_{3}+M\right) \psi=0
$$

In $1+1$ dimensions, the chiral representation of the Dirac gamma matrices for our choice of metric, $\eta^{\alpha \beta}=$ $\operatorname{diag}(-1,1)$, is (compare with Eq. 2.3)

$$
\gamma^{0}=\left[\begin{array}{cc}
0 & 1 \\
-1 & 0
\end{array}\right], \quad \gamma^{3}=\left[\begin{array}{ll}
0 & 1 \\
1 & 0
\end{array}\right]
$$

Consider the change of variables $\left(\xi^{\alpha} \rightarrow x^{a}\right)$ :

$$
t=\tau \cosh \eta, \quad z=\tau \sinh \eta,
$$

with the metric $g_{a b}=\operatorname{diag}\left(-1, \tau^{2}\right)$, and $\sqrt{-g}=\tau$. 


\section{A. Cartesian tetrad representation}

For our change of variables, the Vierbeins are calculated as

$$
\mathrm{V}^{\alpha}{ }_{a}=\left[\begin{array}{ccc}
\cosh \eta & \tau \sinh \eta \\
\sinh \eta & \tau & \cosh \eta
\end{array}\right]
$$

whereas the inverse Vierbeins are

$$
\mathbf{V}^{a}{ }_{\alpha}=\left[\begin{array}{cc}
\cosh \eta & -\sinh \eta \\
-\frac{1}{\tau} \sinh \eta & \frac{1}{\tau} \cosh \eta
\end{array}\right] .
$$

We can now write the Dirac equation as

$$
\left(\tilde{\gamma}^{a} \partial_{a}+M\right) \psi=0,
$$

where the Dirac matrices are given by

$$
\begin{aligned}
& \tilde{\gamma}^{0}=\gamma^{0} \cosh \eta-\gamma^{3} \sinh \eta, \\
& \tilde{\gamma}^{3}=\frac{1}{\tau}\left(-\gamma^{0} \sinh \eta+\gamma^{3} \cosh \eta\right) .
\end{aligned}
$$

Equation (3.4) can be explicitly written as

$$
\left[\begin{array}{cc}
M & \mathcal{T}_{-} \\
-\mathcal{T}_{+} & M
\end{array}\right]\left[\begin{array}{l}
\psi_{1} \\
\psi_{2}
\end{array}\right]=0
$$

where we have introduced the raising and lowering operators

$$
\begin{aligned}
& \mathcal{T}_{-}=\partial_{t}+\partial_{z}=e^{-\eta}\left(\partial_{\tau}+\frac{1}{\tau} \partial_{\eta}\right), \\
& \mathcal{T}_{+}=\partial_{t}-\partial_{z}=e^{\eta}\left(\partial_{\tau}-\frac{1}{\tau} \partial_{\eta}\right),
\end{aligned}
$$

which obey the properties

$$
\begin{aligned}
& \mathcal{T}_{-}\left[e^{\mathrm{i} k_{\eta} \eta} J_{\mathrm{i} k_{\eta}}(M \tau)\right]=+M e^{\left(\mathrm{i} k_{\eta}-1\right) \eta} J_{\mathrm{i} k_{\eta}-1}(M \tau), \\
& \mathcal{T}_{+}\left[e^{\mathrm{i} k_{\eta} \eta} J_{\mathrm{i} k_{\eta}}(M \tau)\right]=-M e^{\left(\mathrm{i} k_{\eta}+1\right) \eta} J_{\mathrm{i} k_{\eta}+1}(M \tau),
\end{aligned}
$$

where $J_{\mathrm{i} k_{\eta}}(M \tau)$ are Bessel functions of complex order (and real argument) 21]. Throughout this paper we use the definitions and properties of the Bessel functions as presented in Ref. 22].

Therefore, we find the amplitudes $\psi_{1}$ and $\psi_{2}$ obey the equations

$$
\begin{aligned}
& \left(\mathcal{T}_{-} \mathcal{T}_{+}+M^{2}\right) \psi_{1}=0 \\
& \left(\mathcal{T}_{+} \mathcal{T}_{-}+M^{2}\right) \psi_{2}=0 .
\end{aligned}
$$

Alternatively we could have "squared" the Dirac equation (3.4) in the usual textbook fashion. This would have doubled the number of solutions and we would have kept only one set such as done in [23]. form

The solutions of the above equations have the general

$$
\psi(\tau, \eta)=\int \mathrm{d} k_{\eta} \psi_{k_{\eta}}(\tau, \eta)
$$

It is necessary [24] to seek a solution $\psi_{k_{\eta}}$ of the form

$$
\psi_{k_{\eta}}=N_{k_{\eta}} e^{\mathrm{i} k_{\eta} \eta}\left[\begin{array}{c}
e^{-\eta / 2} J_{\mathrm{i} k_{\eta}-\frac{1}{2}}(M \tau) \\
-e^{\eta / 2} J_{\mathrm{i} k_{\eta}+\frac{1}{2}}(M \tau)
\end{array}\right] .
$$

Requiring the usual positive/negative-energy boundary conditions [20], we obtain

$$
\psi_{k_{\eta}}^{(+)}=N_{k_{\eta}}^{(+)} e^{\mathrm{i} k_{\eta} \eta}\left[\begin{array}{c}
e^{-\eta / 2} H_{\mathrm{i} k_{\eta}-\frac{1}{2}}^{(2)}(M \tau) \\
-e^{\eta / 2} H_{\mathrm{i} k_{\eta}+\frac{1}{2}}^{(2)}(M \tau)
\end{array}\right],
$$

and

$$
\psi_{k_{\eta}}^{(-)}=N_{k_{\eta}}^{(-)} e^{\mathrm{i} k_{\eta} \eta}\left[\begin{array}{c}
e^{-\eta / 2} H_{\mathrm{i} k_{\eta}-\frac{1}{2}}^{(1)}(M \tau) \\
-e^{\eta / 2} H_{\mathrm{i} k_{\eta}+\frac{1}{2}}^{(1)}(M \tau)
\end{array}\right] .
$$

Then, the canonical quantization of the Dirac field is obtained as

$$
\hat{\psi}(\tau, \eta)=\int \mathrm{d} k_{\eta}\left[\psi_{k_{\eta}}^{(+)}(\tau, \eta) a_{k_{\eta}}+\psi_{k_{\eta}}^{(-)}(\tau, \eta) b_{k_{\eta}}^{\dagger}\right] .
$$

The normalization constants $N_{k_{\eta}}^{( \pm)}$are obtained by requiring the fields $\psi_{k_{\eta}}^{( \pm)}$to satisfy the orthonormality relations

$$
\begin{aligned}
& \left(\bar{\psi}_{k_{\eta}}^{( \pm)}, \psi_{k_{\eta}^{\prime}}^{( \pm)}\right)=\delta\left(k_{\eta}^{\prime}-k_{\eta}\right), \\
& \left(\bar{\psi}_{k_{\eta}}^{(+)}, \psi_{k_{\eta}^{\prime}}^{(-)}\right)=0,
\end{aligned}
$$

where the inner product (2.26) gives

$$
\begin{aligned}
\left(\bar{\psi}_{k_{\eta}}, \psi_{k_{\eta}^{\prime}}\right) & =-\tau \int \mathrm{d} \eta \bar{\psi}_{k_{\eta}} \tilde{\gamma}^{0} \psi_{k_{\eta}^{\prime}} \\
& =\tau \int \mathrm{d} \eta\left[\psi_{1, k_{\eta}}^{(+) \star} \psi_{1, k_{\eta}^{\prime}}^{(+)} e^{\eta}+\psi_{2, k_{\eta}}^{(+) \star} \psi_{2, k_{\eta}^{\prime}}^{(+)} e^{-\eta}\right],
\end{aligned}
$$

with $\bar{\psi}=\psi^{\dagger} \gamma^{0}$. We work out each term separately: 


$$
\begin{gathered}
\psi_{1, k_{\eta}}^{(+) \star} \psi_{1, k_{\eta}^{\prime}}^{(+)} e^{\eta}=N_{k_{\eta}}^{(+) \star} N_{k_{\eta}^{\prime}}^{(+)} \mathrm{i} e^{\mathrm{i}\left(k_{\eta}^{\prime}-k_{\eta}\right) \eta} e^{-\pi k_{\eta}} H_{\mathrm{i} k_{\eta}+\frac{1}{2}}^{(1)} H_{\mathrm{i} k_{\eta}^{\prime}-\frac{1}{2}}^{(2)}, \\
\psi_{2, k_{\eta}}^{(+) \star} \psi_{2, k_{\eta}^{\prime}}^{(+)} e^{-\eta}=-N_{k_{\eta}}^{(+) \star} N_{k_{\eta}^{\prime}}^{(+)} \mathrm{i} e^{\mathrm{i}\left(k_{\eta}^{\prime}-k_{\eta}\right) \eta} e^{-\pi k_{\eta}} H_{\mathrm{i} k_{\eta}-\frac{1}{2}}^{(1)} H_{\mathrm{i} k_{\eta}^{\prime}+\frac{1}{2}}^{(2)}, \\
\psi_{1, k_{\eta}}^{(-) \star} \psi_{1, k_{\eta}^{\prime}}^{(-)} e^{\eta}=N_{k_{\eta}}^{(-) \star} N_{k_{\eta}^{\prime}}^{(-)}(-\mathrm{i}) e^{\mathrm{i}\left(k_{\eta}^{\prime}-k_{\eta}\right) \eta} e^{\pi k_{\eta}} H_{\mathrm{i} k_{\eta}+\frac{1}{2}}^{(2)} H_{\mathrm{i} k_{\eta}^{\prime}-\frac{1}{2}}^{(1)}, \\
\psi_{2, k_{\eta}}^{(-) \star} \psi_{2, k_{\eta}^{\prime}}^{(-)} e^{-\eta}=-N_{k_{\eta}}^{(-) \star} N_{k_{\eta}^{\prime}}^{(-)}(-\mathrm{i}) e^{\mathrm{i}\left(k_{\eta}^{\prime}-k_{\eta}\right) \eta} e^{\pi k_{\eta}} H_{\mathrm{i} k_{\eta}-\frac{1}{2}}^{(2)} H_{\mathrm{i} k_{\eta}^{\prime}+\frac{1}{2}}^{(1)},
\end{gathered}
$$

where we have used the properties of the Hankel functions of real argument, $x$ 22]

$$
\begin{aligned}
H_{\nu}^{(1,2) \star}(x) & =H_{\nu^{\star}}^{(2,1)}(x), \\
H_{-\nu}^{(1,2)}(x) & =e^{(+,-) \mathrm{i} \pi \nu} H_{\nu}^{(1,2)}(x) .
\end{aligned}
$$

We will also use the Wronskian identity [22]:

$$
H_{\mathrm{i} k}^{(1)}(x) H_{\mathrm{i} k-1}^{(2)}(x)-H_{\mathrm{i} k-1}^{(1)}(x) H_{\mathrm{i} k}^{(2)}(x)=-\frac{4 \mathrm{i}}{\pi x} .
$$

We note that by performing the integral in (3.21) we obtain

$$
\int \mathrm{d} \eta e^{\mathrm{i}\left(k^{\prime}-k\right) \eta}=2 \pi \delta\left(k^{\prime}-k\right) .
$$

Then, we find the normalization constants must satisfy the conditions

$$
1=N_{k_{\eta}}^{( \pm) \star} N_{k_{\eta}}^{( \pm)} e^{\mp \pi k_{\eta}} \frac{8}{M} .
$$

We pick the normalization constants as:

$$
N_{k_{\eta}}^{( \pm)}=\sqrt{\frac{M}{8}} e^{ \pm \pi k_{\eta} / 2}
$$

Therefore, we find that the positive/negative energy solutions of (3.4) are

$$
\psi_{k_{\eta}}^{(+)}=\sqrt{\frac{M}{8}} e^{\pi k_{\eta} / 2} e^{\mathrm{i} k_{\eta} \eta}\left[\begin{array}{c}
e^{-\eta / 2} H_{\mathrm{i} k_{\eta}-\frac{1}{2}}^{(2)}(M \tau) \\
-e^{\eta / 2} H_{\mathrm{i} k_{\eta}+\frac{1}{2}}^{(2)}(M \tau)
\end{array}\right],
$$

and

$$
\psi_{k_{\eta}}^{(-)}=\sqrt{\frac{M}{8}} e^{-\pi k_{\eta} / 2} e^{\mathrm{i} k_{\eta} \eta}\left[\begin{array}{c}
e^{-\eta / 2} H_{\mathrm{i} k_{\eta}-\frac{1}{2}}^{(1)}(M \tau) \\
-e^{\eta / 2} H_{\mathrm{i} k_{\eta}+\frac{1}{2}}^{(1)}(M \tau)
\end{array}\right] .
$$

\section{B. Diagonal tetrad representation}

We begin with Eq. (3.4) and introduce the similarity transformation

$$
S_{\tau}=\cosh \frac{\eta}{2}-\gamma^{0} \gamma^{3} \sinh \frac{\eta}{2}=\exp \left(-\frac{\eta}{2} \gamma^{0} \gamma^{3}\right),
$$

such that

$$
S_{\tau}^{-1} \tilde{\gamma}^{a} S_{\tau}=\bar{\gamma}^{a},
$$

with

$$
\bar{\gamma}^{0}=\gamma^{0}, \quad \bar{\gamma}^{3}=\frac{1}{\tau} \gamma^{3} .
$$

The diagonal (rotating) Vierbein representation is identified as

$$
\begin{aligned}
\overline{\mathrm{V}}^{\alpha}{ }_{a} & =\operatorname{diag}(1, \tau), \\
\overline{\mathbf{V}}^{a}{ }_{\alpha} & =\operatorname{diag}\left(1, \frac{1}{\tau}\right) .
\end{aligned}
$$

The Dirac equation in the diagonal tetrad representation is

$$
\left(\bar{\gamma}^{a} \nabla_{a}+M\right) \psi_{[\mathrm{d}]}=0
$$

where we have introduced the diagonal Dirac wavefunction

$$
\psi_{[\mathrm{d}]}=S_{\tau}^{-1} \psi .
$$

The identification process described in Sec. Il leads to the nonzero Christoffel symbols

$$
\begin{array}{r}
\Gamma_{\eta \eta}^{\tau}=\tau, \\
\Gamma_{\tau \eta}^{\eta}=\Gamma_{\eta \tau}^{\eta}=\frac{1}{\tau},
\end{array}
$$

the spinor connections

$$
\Gamma_{\eta}=\frac{1}{2} \gamma^{0} \gamma^{3}, \quad \Gamma_{\tau}=0,
$$

and the covariant derivatives

$$
\nabla_{\tau}=\partial_{\tau}, \quad \nabla_{\eta}=\partial_{\eta}-\frac{1}{2} \gamma^{0} \gamma^{3} .
$$

Finally, the Dirac equation becomes

$$
\left[\gamma^{0}\left(\partial_{\tau}+\frac{1}{2 \tau}\right)+\gamma^{3} \frac{1}{\tau} \partial_{\eta}+M\right] \psi_{[\mathrm{d}]}=0,
$$

and has a solution of the form

$$
\psi_{k_{\eta}[\mathrm{d}]}=N_{k_{\eta}} e^{\mathrm{i} k_{\eta} \eta}\left[\begin{array}{c}
J_{\mathrm{i} k_{\eta}-\frac{1}{2}}(M \tau) \\
-J_{\mathrm{i} k_{\eta}+\frac{1}{2}}(M \tau)
\end{array}\right] .
$$


We find positive- and negative-energy solutions obeying the relations (3.19), as

$$
\psi_{k_{\eta}[\mathrm{d}]}^{(+)}=\sqrt{\frac{M}{8}} e^{\pi k_{\eta} / 2} e^{\mathrm{i} k_{\eta} \eta}\left[\begin{array}{c}
H_{\mathrm{i} k_{\eta}-\frac{1}{2}}^{(2)}(M \tau) \\
-H_{\mathrm{i} k_{\eta}+\frac{1}{2}}^{(2)}(M \tau)
\end{array}\right],
$$

and

$$
\psi_{k_{\eta}[\mathrm{d}]}^{(-)}=\sqrt{\frac{M}{8}} e^{-\pi k_{\eta} / 2} e^{\mathrm{i} k_{\eta} \eta}\left[\begin{array}{c}
H_{\mathrm{i} k_{\eta}-\frac{1}{2}}^{(1)}(M \tau) \\
-H_{\mathrm{i} k_{\eta}+\frac{1}{2}}^{(1)}(M \tau)
\end{array}\right] .
$$

Finally, the Dirac fields in the Cartesian and diagonal tetrad representations are related via the transformation (3.40). Indeed, with our choice of gamma matrices, we obtain

$$
\gamma^{0} \gamma^{3}=\left[\begin{array}{cc}
1 & 0 \\
0 & -1
\end{array}\right]
$$

which gives the explicit form of the similarity transformation (3.34)

$$
S_{\tau}=\left[\begin{array}{cc}
e^{-\eta / 2} & 0 \\
0 & e^{\eta / 2}
\end{array}\right] .
$$

We immediately verify that the Dirac solutions (3.32) and (3.33) are obtained from (3.47) and (3.48) as

$$
\psi_{k_{\eta}}^{( \pm)}=S_{\tau} \psi_{k_{\eta}[\mathrm{d}]}^{( \pm)} .
$$

\section{CYLINDRICAL COORDINATES IN 3+1 DIMENSIONS}

The canonical quantization of the Dirac equation in cylindrical coordinates has been studied in detail by Balantekin and DeWeerd [15] using the eigenstates of the transverse helicity operators to obtain a complete set of solutions. Here, for uniformity of presentation we will use the tetrad formalism to obtain a related decomposition in terms of Bessel functions of $1 / 2$ integer order. Consider the change of variables $\left(\xi^{\alpha} \rightarrow x^{a}\right)$ :

$$
\begin{aligned}
& t=t, \quad z=z, \\
& x=\rho \cos \theta, \quad y=\rho \sin \theta .
\end{aligned}
$$

with the metric $g_{a b}=\operatorname{diag}\left(-1,1, \rho^{2}, 1\right)$, and $\sqrt{-g}=\rho$.

\section{A. Cartesian tetrad representation}

The Vierbeins in fixed-tetrad representation are calculated as

$$
\mathrm{V}_{a}^{\alpha}=\left[\begin{array}{cccc}
1 & 0 & 0 & 0 \\
0 & \cos \theta & -\rho \sin \theta & 0 \\
0 & \sin \theta & \rho \cos \theta & 0 \\
0 & 0 & 0 & 1
\end{array}\right]
$$

whereas the inverse Vierbeins are obtained as

$$
\mathbf{V}_{\alpha}^{a}=\left[\begin{array}{cccc}
1 & 0 & 0 & 0 \\
0 & \cos \theta & \sin \theta & 0 \\
0 & -\frac{1}{\rho} \sin \theta & \frac{1}{\rho} \cos \theta & 0 \\
0 & 0 & 0 & 1
\end{array}\right] .
$$

The Dirac equation becomes

$$
\left(\tilde{\gamma}^{a} \partial_{a}+M\right) \psi=0,
$$

with the gamma matrices, $\tilde{\gamma}^{a}=\mathbf{V}^{a}{ }_{\alpha} \gamma^{\alpha}$, defined as

$$
\begin{aligned}
& \tilde{\gamma}^{0}=\gamma^{0}, \quad \tilde{\gamma}^{3}=\gamma^{3}, \\
& \tilde{\gamma}^{1}=\gamma^{1} \cos \theta+\gamma^{2} \sin \theta, \\
& \tilde{\gamma}^{2}=\frac{1}{\rho}\left(-\gamma^{1} \sin \theta+\gamma^{2} \cos \theta\right) .
\end{aligned}
$$

Following Villalba [10], we write Eq. (4.2) as

$$
\left(\mathcal{K}_{1}+\mathcal{K}_{2}\right) \Phi=0, \quad \Phi=\mathrm{i} \gamma^{1} \gamma^{2} \psi
$$

where $\mathcal{K}_{1}$ and $\mathcal{K}_{2}$ are the first-order differential operators

$$
\begin{aligned}
& \mathcal{K}_{1}(t, z)=\left(\tilde{\gamma}^{0} \partial_{t}+\tilde{\gamma}^{3} \partial_{z}\right) \mathrm{i} \gamma^{1} \gamma^{2}, \\
& \mathcal{K}_{2}(\rho, \theta)=\left(\tilde{\gamma}^{1} \partial_{\rho}+\tilde{\gamma}^{2} \partial_{\theta}+M\right) \mathrm{i} \gamma^{1} \gamma^{2},
\end{aligned}
$$

and

$$
\mathrm{i} \gamma^{1} \gamma^{2}=-\left[\begin{array}{cc}
\sigma_{3} & 0 \\
0 & \sigma_{3}
\end{array}\right]
$$

Since the operators $\mathcal{K}_{1}$ and $\mathcal{K}_{2}$ commute 25$],\left[\mathcal{K}_{1}, \mathcal{K}_{2}\right]=0$, we can write the eigenvalue equations

$$
\mathcal{K}_{1} \Phi=-\mathcal{K}_{2} \Phi=-\lambda \Phi .
$$

We will find the solution of Eq. (4.6) has the form

$$
\Phi(t, \rho, \theta, z)=\int k_{\perp} \mathrm{d} k_{\perp} \Phi^{k_{\perp}}(t, \rho, \theta, z),
$$

where $\lambda^{2}-M^{2}=k_{\perp}^{2}$.

a. $\mathcal{K}_{2}$ sector. The structure of the amplitudes $\Phi^{k_{\perp}}$ is established by solving the eigenvector problem for the operator $\mathcal{K}_{2}$. We have

$$
\left(\mathcal{K}_{2}-\lambda\right) \Phi^{k_{\perp}}(\rho, \theta)=0,
$$

or

$$
\left(\mathrm{i} \gamma^{2} \partial_{x}-\mathrm{i} \gamma^{1} \partial_{y}+\mathrm{i} \gamma^{1} \gamma^{2} M-\lambda\right) \Phi^{k_{\perp}}=0,
$$

which gives

$$
\left[\begin{array}{cccc}
-\lambda_{+} & 0 & 0 & \mathcal{P}_{-} \\
0 & -\lambda_{-} & -\mathcal{P}_{+} & 0 \\
0 & \mathcal{P}_{-} & -\lambda_{+} & 0 \\
-\mathcal{P}_{+} & 0 & 0 & -\lambda_{-}
\end{array}\right]\left[\begin{array}{l}
\Phi^{k_{\perp} ; 1}(\rho, \theta) \\
\Phi^{k_{\perp} ; 2}(\rho, \theta) \\
\Phi^{k_{\perp} ; 3}(\rho, \theta) \\
\Phi^{k_{\perp} ; 4}(\rho, \theta)
\end{array}\right]=0,
$$


where we have introduced the notations $\lambda_{ \pm}=\lambda \pm M$. The raising and lowering operators are defined as

$$
\begin{aligned}
& \mathcal{P}_{-}=\partial_{x}-\mathrm{i} \partial_{y}=e^{-\mathrm{i} \theta}\left(\partial_{\rho}-\frac{\mathrm{i}}{\rho} \partial_{\theta}\right), \\
& \mathcal{P}_{+}=\partial_{x}+\mathrm{i} \partial_{y}=e^{\mathrm{i} \theta}\left(\partial_{\rho}+\frac{\mathrm{i}}{\rho} \partial_{\theta}\right),
\end{aligned}
$$

with the properties

$$
\begin{aligned}
& \mathcal{P}_{-}\left[e^{\mathrm{i} m \theta} J_{m}\left(k_{\perp} \rho\right)\right]=+k_{\perp}\left[e^{\mathrm{i}(m-1) \theta} J_{m-1}\left(k_{\perp} \rho\right)\right] \\
& \mathcal{P}_{+}\left[e^{\mathrm{i} m \theta} J_{m}\left(k_{\perp} \rho\right)\right]=-k_{\perp}\left[e^{\mathrm{i}(m+1) \theta} J_{m+1}\left(k_{\perp} \rho\right)\right] .
\end{aligned}
$$

The system of equations (4.14) decouple, and we obtain

$$
\begin{aligned}
& {\left[\mathcal{P}_{+} \mathcal{P}_{-}+\lambda_{+} \lambda_{-}\right] \Phi^{k_{\perp} ; 2[4]}(\rho, \theta)=0,} \\
& {\left[\mathcal{P}_{-} \mathcal{P}_{+}+\lambda_{+} \lambda_{-}\right] \Phi^{k_{\perp} ; 3[1]}(\rho, \theta)=0 .}
\end{aligned}
$$

The spinor $\Phi^{k_{\perp}}(\rho, \theta)$ has the form

$$
\Phi^{k_{\perp}}(\rho, \theta)=\sum_{m=-\infty}^{\infty} \Phi_{m}^{k_{\perp}}(\rho, \theta)
$$

with

$$
\Phi_{m}^{k_{\perp}}(\rho, \theta)=e^{\mathrm{i} m \theta}\left[\begin{array}{r}
A^{k_{\perp}} e^{-\mathrm{i} \theta / 2} J_{m-\frac{1}{2}}\left(k_{\perp} \rho\right) \\
B^{k_{\perp}} e^{\mathrm{i} \theta / 2} J_{m+\frac{1}{2}}\left(k_{\perp} \rho\right) \\
C^{k_{\perp}} e^{-\mathrm{i} \theta / 2} J_{m-\frac{1}{2}}\left(k_{\perp} \rho\right) \\
D^{k_{\perp}} e^{\mathrm{i} \theta / 2} J_{m+\frac{1}{2}}\left(k_{\perp} \rho\right)
\end{array}\right],
$$

and obtain the constraints

$$
\begin{aligned}
D^{k_{\perp}} & =\frac{\lambda_{+}}{k_{\perp}} A^{k_{\perp}}=\frac{k_{\perp}}{\lambda_{-}} A^{k_{\perp}}, \\
B^{k_{\perp}} & =\frac{k_{\perp}}{\lambda_{-}} C^{k_{\perp}}=\frac{\lambda_{+}}{k_{\perp}} C^{k_{\perp}} .
\end{aligned}
$$

We also find

$$
\lambda_{+} \lambda_{-}=\lambda^{2}-M^{2}=k_{\perp}^{2},
$$

which gives

$$
\lambda^{2}=\omega_{\perp}^{2}=M^{2}+k_{\perp}^{2}, \text { or } \lambda= \pm \omega_{\perp} .
$$

Corresponding to the two eigenvalues, $\lambda= \pm \omega_{\perp}$, we have two linearly-independent eigenvectors

$$
\Phi_{m}^{k_{\perp} ; s}(\rho, \theta)=e^{\mathrm{i} m \theta}\left[\begin{array}{c}
A^{k_{\perp}} e^{-\mathrm{i} \theta / 2} J_{m-\frac{1}{2}}\left(k_{\perp} \rho\right) \\
\frac{k_{\perp}}{\lambda_{-}} C^{k_{\perp}} e^{\mathrm{i} \theta / 2} J_{m+\frac{1}{2}}\left(k_{\perp} \rho\right) \\
C^{k_{\perp}} e^{-\mathrm{i} \theta / 2} J_{m-\frac{1}{2}}\left(k_{\perp} \rho\right) \\
\frac{k_{\perp}}{\lambda_{-}} A^{k_{\perp}} e^{\mathrm{i} \theta / 2} J_{m+\frac{1}{2}}\left(k_{\perp} \rho\right)
\end{array}\right],
$$

with $\lambda=s \omega_{\perp}$, and $s= \pm 1$. b. $\quad \mathcal{K}_{1}$ sector. The amplitudes $A^{k_{\perp}}$ and $C^{k_{\perp}}$ are determined by solving the eigenvalue equation for the operator $\mathcal{K}_{1}$, i.e.

$$
\left(\mathcal{K}_{1}+\lambda\right) \Phi_{m}^{k_{\perp} ; s}(t, z)=0,
$$

or

$$
\left[\mathrm{i} \gamma^{0} \gamma^{1} \gamma^{2} \partial_{t}+\mathrm{i} \gamma^{3} \gamma^{1} \gamma^{2} \partial_{z}+\lambda\right] \Phi_{m}^{k_{\perp} ; s}(t, z)=0 .
$$

Once again using the representation (2.3) of the Dirac gamma matrices, we obtain the anticommuting products of gamma matrices:

$$
\mathrm{i} \gamma^{0} \gamma^{1} \gamma^{2}=\left[\begin{array}{cc}
0 & -\sigma_{3} \\
\sigma_{3} & 0
\end{array}\right], \quad \mathrm{i} \gamma^{3} \gamma^{1} \gamma^{2}=\left[\begin{array}{cc}
0 & -1 \\
-1 & 0
\end{array}\right] .
$$

Since $\partial_{t}$ and $\partial_{z}$ commute with the above equation, we can also Fourier transform the Dirac field in $t$ and $z$. We introduce the field as

$$
\Phi_{m}^{k_{\perp} ; s}=\int \mathrm{d} E e^{\mathrm{i} E t} \int \mathrm{d} k_{z} e^{-\mathrm{i} k_{z} z} \Phi_{m, k_{z} ; E}^{k_{\perp} ; s},
$$

which gives

$$
\left\{\left[\begin{array}{cc}
0 & -\sigma_{3} \\
\sigma_{3} & 0
\end{array}\right](\mathrm{i} E)+\left[\begin{array}{cc}
0 & -1 \\
-1 & 0
\end{array}\right]\left(-\mathrm{i} k_{z}\right)+\lambda\right\} \Phi_{m, k_{z} ; E}^{k_{\perp}}=0 .
$$

Eq. (4.32) is equivalent to

$$
\left[\begin{array}{cc}
\lambda & \mathrm{i}\left(k_{z}-E\right) \\
\mathrm{i}\left(k_{z}+E\right) & \lambda
\end{array}\right]\left[\begin{array}{c}
A_{k_{z}, E}^{k_{\perp}} \\
C_{k_{z}, E}^{k, E}
\end{array}\right]=0 .
$$

For a nontrivial solution, we require the determinant to be zero, which gives the usual dispersion relations

$$
E= \pm \sqrt{\omega_{\perp}^{2}+k_{z}^{2}}= \pm \sqrt{M^{2}+\mathbf{k}^{2}} .
$$

We obtain

$$
C_{k_{z}, E}^{k_{\perp}}=\frac{\mathrm{i} \lambda}{k_{z}-E} A_{k_{z}, E}^{k_{\perp}}=\frac{-\mathrm{i}\left(k_{z}+E\right)}{\lambda} A_{k_{z}, E}^{k_{\perp}} .
$$

Hence, the solution to the free-field Dirac equation can be written as

$$
\begin{aligned}
& \psi_{m, k_{z} ; E}^{k_{\perp} ; s}(t, \rho, \theta, z)=\mathrm{i} \gamma^{1} \gamma^{2} \Phi_{m, k_{z} ; E}^{k_{\perp} ; s}(t, \rho, \theta, z) \\
& =N_{k_{z}, E}^{k_{\perp} ; s} e^{\mathrm{i}\left(m \theta-k_{z} z\right)}\left[\begin{array}{r}
-e^{-\mathrm{i} \theta / 2} J_{m-\frac{1}{2}}\left(k_{\perp} \rho\right) \\
\frac{k_{\perp}}{\lambda-M} \frac{\mathrm{i} \lambda}{k_{z}-E} e^{\mathrm{i} \theta / 2} J_{m+\frac{1}{2}}\left(k_{\perp} \rho\right) \\
-\frac{\mathrm{i} \lambda}{k_{z}-E} e^{-\mathrm{i} \theta / 2} J_{m-\frac{1}{2}}\left(k_{\perp} \rho\right) \\
\frac{k \perp}{\lambda-M} e^{\mathrm{i} \theta / 2} J_{m+\frac{1}{2}}\left(k_{\perp} \rho\right)
\end{array}\right] .
\end{aligned}
$$

The canonical quantization of the Dirac field in cylindrical coordinates is obtained as

$$
\begin{aligned}
\hat{\psi}(t, \rho, \theta, z)= & \int k_{\perp} \mathrm{d} k_{\perp} \int \mathrm{d} k_{z} \sum_{m=-\infty}^{\infty} \sum_{s} \\
& \times\left[\psi_{m, k_{z}}^{k_{\perp}(s)} a_{m, k_{z}}^{k_{\perp} ; s}+\psi_{m, k_{z}}^{k_{\perp} ; s(-)} b_{m, k_{z}}^{k_{\perp} \dagger}\right] .
\end{aligned}
$$


where $\psi_{m, k_{z}}^{k_{\perp}( \pm)}$ correspond to the positive and negative energy solutions, respectively. The normalization constants $N_{k_{z},( \pm)|E|}^{k_{\perp} ; s}$ are obtained by requiring the fields $\psi_{m, k_{z}}^{k_{\perp} ; s( \pm)}$ to satisfy the orthonormality relations

$$
\begin{aligned}
& \left(\bar{\psi}_{m, k_{z}}^{k_{\perp} ; s( \pm)}, \psi_{m^{\prime}, k_{z}^{\prime}}^{k_{\perp}^{\prime} ;( \pm)}\right)=\frac{\delta\left(k_{\perp}^{\prime}-k_{\perp}\right)}{k_{\perp}} \delta\left(k_{z}^{\prime}-k_{z}\right) \delta_{m^{\prime} m} \delta_{s^{\prime} s}, \\
& \left(\bar{\psi}_{m, k_{z}}^{k_{\perp} ; s(+)}, \psi_{m^{\prime}, k_{z}^{\prime}}^{k_{1}^{\prime} ; s^{\prime}(-)}\right)=0 .
\end{aligned}
$$

where we have introduced the inner product

$$
\begin{gathered}
\left(\bar{\psi}_{m, k_{z}}^{k_{\perp} ; s}, \psi_{m_{\perp}^{\prime}, k_{z}^{\prime}}^{k_{\perp}^{\prime} ; s^{\prime}}\right)=-\int \rho \mathrm{d} \rho \int \mathrm{d} \theta \int \mathrm{d} z \bar{\psi}_{m, k_{z}}^{k_{\perp} ; s} \tilde{\gamma}^{0} \psi_{m^{\prime}, k_{z}^{\prime}}^{k_{\perp}^{\prime} ; s^{\prime}} \\
=\int \rho \mathrm{d} \rho \int \mathrm{d} \theta \int \mathrm{d} z \psi_{m, k_{z}}^{k_{\perp} ; s \dagger} \psi_{m^{\prime}, k_{z}^{\prime}}^{k_{\perp}^{\prime} ;}
\end{gathered}
$$

Using the identities

$$
\begin{aligned}
2 \pi \delta_{m^{\prime} m} & =\int \mathrm{d} \theta e^{\mathrm{i}\left(m^{\prime}-m\right) \theta}, \\
2 \pi \delta\left(k_{z}^{\prime}-k_{z}\right) & =\int \mathrm{d} z e^{\mathrm{i}\left(k_{z}^{\prime}-k_{z}\right) z}, \\
\frac{\delta\left(k_{\perp}^{\prime}-k_{\perp}\right)}{k_{\perp}} & =\int \rho \mathrm{d} \rho J_{\nu}\left(k_{\perp}^{\prime} \rho\right) J_{\nu}\left(k_{\perp} \rho\right),
\end{aligned}
$$

we obtain the normalization constant

$$
N_{k_{z}, E}^{k_{\perp} ; s}=\frac{1}{2 \pi} \sqrt{\frac{E-k_{z}}{2 E}} \sqrt{\frac{\lambda-M}{2 \lambda}},
$$

for either positive or negative values of $E$ and $\lambda$. This completes our derivation.

\section{B. Diagonal tetrad representation}

In cylindrical coordinates, we introduce the similarity transformation

$$
S_{\rho}=\cos \frac{\theta}{2}-\gamma^{1} \gamma^{2} \sin \frac{\theta}{2}=\exp \left(-\frac{\theta}{2} \gamma^{1} \gamma^{2}\right),
$$

such that

$$
S_{\rho}^{-1} \tilde{\gamma}^{a} S_{\rho}=\bar{\gamma}^{a}
$$

where

$$
\bar{\gamma}^{0}=\gamma^{0}, \quad \bar{\gamma}^{1}=\gamma^{1}, \quad \bar{\gamma}^{2}=\frac{1}{\rho} \gamma^{2}, \quad \bar{\gamma}^{3}=\gamma^{3} .
$$

The diagonal Vierbein representation, $\bar{\gamma}^{\alpha}=\overline{\mathbf{V}}^{a}{ }_{\alpha} \gamma^{\alpha}$, is introduced as

$$
\begin{aligned}
\overline{\mathrm{V}}_{a}^{\alpha} & =\operatorname{diag}(1,1, \rho, 1), \\
\overline{\mathbf{V}}_{\alpha}^{a} & =\operatorname{diag}\left(1,1, \frac{1}{\rho}, 1\right),
\end{aligned}
$$

and the Dirac equation can be written as

$$
\left(\bar{\gamma}^{a} \nabla_{a}+M\right) \psi_{[\mathrm{d}]}=0,
$$

where we have introduced the diagonal Dirac wave function

$$
\psi_{[\mathrm{d}]}=S_{\rho}^{-1} \psi .
$$

In the cylindrical system of coordinates, the covariant derivatives

$$
\nabla_{a}=S_{\rho}^{-1} \partial_{a} S_{\rho}=\partial_{a}-\Gamma_{a},
$$

Here, the spinor connection $\Gamma_{a}$,

$$
\Gamma_{\theta}=\frac{1}{2} \gamma^{1} \gamma^{2}, \quad \Gamma_{t}=\Gamma_{\rho}=\Gamma_{z}=0,
$$

are obtained using the (nonzero) Christoffel symbols

$$
\begin{aligned}
\Gamma_{\theta \theta}^{\rho} & =-\rho, \\
\Gamma_{\rho \theta}^{\theta}=\Gamma_{\theta \rho}^{\theta} & =\frac{1}{\rho} .
\end{aligned}
$$

The covariant derivatives are obtained as

$$
\begin{aligned}
\nabla_{t}=\partial_{t}, & \nabla_{\rho}=\partial_{\rho}, \\
\nabla_{\theta}=\partial_{\theta}-\frac{1}{2} \gamma^{1} \gamma^{2}, & \nabla_{z}=\partial_{z} .
\end{aligned}
$$

With the above definitions, the Dirac equation (4.50) can be written as

$$
\left[\gamma^{0} \partial_{t}+\gamma^{1}\left(\partial_{\rho}+\frac{1}{2 \rho}\right)+\gamma^{2} \frac{1}{\rho} \partial_{\theta}+\gamma^{3} \partial_{z}+M\right] \psi_{[\mathrm{d}]}=0 .
$$

Following the same approach to the separation of variables as in the case of the Cartesian tetrad representation, with the difference that now the operator $\mathcal{K}_{2}$ is

$$
\mathcal{K}_{2}(\rho, \theta)=\left[\gamma^{1}\left(\partial_{\rho}+\frac{1}{2 \rho}\right)+\gamma^{2} \frac{1}{\rho} \partial_{\theta}+M\right] \mathrm{i} \gamma^{1} \gamma^{2} .
$$

We find that the solution of Eq. (4.57) has the form

$$
\psi_{E[d]}=\int k_{\perp} \mathrm{d} k_{\perp} \int \mathrm{d} k_{z} \sum_{m=-\infty}^{\infty} \sum_{s} \psi_{m, k_{z} ; E[d]}^{k_{\perp} ;},
$$

where the function $\psi_{m, k_{z} ; E}^{k_{\perp} ; s[d]}$ is given by

$$
\begin{aligned}
& \psi_{m, k_{z} ; E[d]}^{k_{\perp}}(t, \rho, \theta, z)=\mathrm{i} \gamma^{1} \gamma^{2} \Phi_{m, k_{z} ; E[d]}^{k_{\perp} ; s}(t, \rho, \theta, z) \\
& =N_{k_{z}, E}^{k_{\perp} ; s} e^{\mathrm{i}\left(m \theta-k_{z} z\right)}\left[\begin{array}{r}
-J_{m-\frac{1}{2}}\left(k_{\perp} \rho\right) \\
\frac{k_{\perp}}{\lambda-M} \frac{\mathrm{i} \lambda}{k_{z}-E} J_{m+\frac{1}{2}}\left(k_{\perp} \rho\right) \\
-\frac{\mathrm{i} \lambda}{k_{z}-E} J_{m-\frac{1}{2}}\left(k_{\perp} \rho\right) \\
\frac{k_{\perp}}{\lambda-M} J_{m+\frac{1}{2}}\left(k_{\perp} \rho\right)
\end{array}\right],
\end{aligned}
$$


where $N_{k_{z}, E}^{k_{\perp} ; s}$ is given by Eq. (4.44). The Dirac fields in the Cartesian and diagonal tetrad representations (see Eqs. (4.36) and (4.60), respectively), respectively are related via the similarity transformation introduced in Eq. (4.51). As advertised, we obtain

$$
S_{\rho}=\left[\begin{array}{cccc}
e^{-\mathrm{i} \theta / 2} & 0 & 0 & 0 \\
0 & e^{\mathrm{i} \theta / 2} & 0 & 0 \\
0 & 0 & e^{-\mathrm{i} \theta / 2} & 0 \\
0 & 0 & 0 & e^{\mathrm{i} \theta / 2}
\end{array}\right]
$$

which leads to

$$
\psi_{m, k_{z} ; E}^{k_{\perp} ; s}=S_{\rho} \psi_{m, k_{z} ; E[d]}^{k_{\perp} ; s} .
$$

\section{BOOST-INVARIANT COORDINATES WITH AZIMUTHAL SYMMETRY}

Consider the change of variables $\left(\xi^{\alpha} \rightarrow x^{a}\right)$ :

$$
\begin{aligned}
& t=\tau \cosh \eta, \quad z=\tau \sinh \eta, \\
& x=\rho \cos \theta, \quad y=\rho \sin \theta,
\end{aligned}
$$

which gives the metric $g_{a b}=\operatorname{diag}\left(-1,1, \rho^{2}, \tau^{2}\right)$, and $\sqrt{-g}=\tau \rho$.

Since the $(x, y)$ and $(t, z)$ subspaces do not interact, the transformations we need are indeed very simple. The key ingredients are:

- Cartesian Vierbeins:

$$
\mathrm{V}_{a}^{\alpha}=\left[\begin{array}{cccc}
\cosh \eta & 0 & 0 & \tau \sinh \eta \\
0 & \cos \theta & -\rho \sin \theta & 0 \\
0 & \sin \theta & \rho \cos \theta & 0 \\
\sinh \eta & 0 & 0 & \tau \cosh \eta
\end{array}\right],
$$

and

$$
\mathbf{V}_{\alpha}^{a}=\left[\begin{array}{cccc}
\cosh \eta & 0 & 0 & -\sinh \eta \\
0 & \cos \theta & \sin \theta & 0 \\
0 & -\frac{1}{\rho} \sin \theta & \frac{1}{\rho} \cos \theta & 0 \\
-\frac{1}{\tau} \sinh \eta & 0 & 0 & \frac{1}{\tau} \cosh \eta
\end{array}\right] .
$$

- gamma matrices in the fixed-tetrad representation:

$$
\begin{aligned}
& \tilde{\gamma}^{0}=\gamma^{0} \cosh \eta-\gamma^{3} \sinh \eta \\
& \tilde{\gamma}^{3}=\frac{1}{\tau}\left(-\gamma^{0} \sinh \eta+\gamma^{3} \cosh \eta\right) \\
& \tilde{\gamma}^{1}=\gamma^{1} \cos \theta+\gamma^{2} \sin \theta \\
& \tilde{\gamma}^{2}=\frac{1}{\rho}\left(-\gamma^{1} \sin \theta+\gamma^{2} \cos \theta\right) .
\end{aligned}
$$

- similarity transformation: $S=S_{\tau} S_{\rho}$. Note that the individual similarity transformations commute with each other.
- diagonal Vierbeins:

$$
\begin{aligned}
\overline{\mathrm{V}}^{\alpha}{ }_{a} & =\operatorname{diag}(1,1, \rho, \tau), \\
\overline{\mathbf{V}}^{a}{ }_{\alpha} & =\operatorname{diag}\left(1,1, \frac{1}{\rho}, \frac{1}{\tau}\right),
\end{aligned}
$$

- gamma matrices in the rotating-tetrad representation: $\bar{\gamma}^{a}=S^{-1} \tilde{\gamma}^{a} S$, where

$$
\bar{\gamma}^{0}=\gamma^{0}, \quad \bar{\gamma}^{1}=\gamma^{1}, \quad \bar{\gamma}^{2}=\frac{1}{\rho} \gamma^{2}, \quad \bar{\gamma}^{3}=\frac{1}{\tau} \gamma^{3} .
$$

- diagonal Dirac field:

$$
\psi_{[\mathrm{d}]}=S^{-1} \psi=S_{\tau}^{-1} S_{\rho}^{-1} \psi .
$$

- covariant derivatives:

$$
\begin{array}{r}
\nabla_{\tau}=\partial_{\tau}, \quad \nabla_{\rho}=\partial_{\rho}, \\
\nabla_{\theta}=\partial_{\theta}-\frac{1}{2} \gamma^{1} \gamma^{2}, \quad \nabla_{\eta}=\partial_{\eta}-\frac{1}{2} \gamma^{0} \gamma^{3} .
\end{array}
$$

\section{A. Cartesian tetrad representation}

The Dirac equation

$$
\left(\tilde{\gamma}^{a} \partial_{a}+M\right) \psi=0
$$

is solved using the separation of variables procedure outlined before, see Eqs. (4.6), (4.7) and (4.8). The modified Dirac field $\Phi(\tau, \rho, \theta, \eta)$ has the form (4.11), and we solve the eigenvalue problems defined in Eq. (4.10). Again, since there is no interaction between the $(x, y)$ and $(t, z)$ subspaces, it follows that the $(\rho, \theta)$ structure of $\Phi(\tau, \rho, \theta, \eta)$ remains the same as in (4.27), and we only need to concern ourselves only with solving the eigenvalue problem for the operator $\mathcal{K}_{1}$ in the new set of coordinates, $(\tau, \eta)$. Explicitly, we seek the amplitudes $A_{k_{\eta}}^{k_{\perp}}(\tau)$ and $C_{k_{\eta}}^{k_{\perp}}(\tau)$ required by (4.27).

Consider the eigenvalue problem

$$
\left(\mathcal{K}_{1}+\lambda\right) \Phi^{k_{\perp}}(\tau)=0 .
$$

Using the raising and lowering operators defined in (3.9) and (3.8), respectively, the above equation reads

$$
\left[\begin{array}{cccc}
\lambda & 0 & -\mathcal{T}_{-} & 0 \\
0 & \lambda & 0 & \mathcal{T}_{+} \\
\mathcal{T}_{+} & 0 & \lambda & 0 \\
0 & -\mathcal{T}_{-} & 0 & \lambda
\end{array}\right]\left[\begin{array}{c}
A_{k_{\eta}}^{k_{\perp}}(\tau) e^{-\mathrm{i} \theta / 2} J_{m-\frac{1}{2}}\left(k_{\perp} \rho\right) \\
\frac{k_{\perp}}{\lambda_{-}} C_{k_{\eta}}^{k_{\perp}}(\tau) e^{\mathrm{i} \theta / 2} J_{m+\frac{1}{2}}\left(k_{\perp} \rho\right) \\
C_{k_{\eta}}^{k_{\perp}}(\tau) e^{-\mathrm{i} \theta / 2} J_{m-\frac{1}{2}}\left(k_{\perp} \rho\right) \\
\frac{k_{\perp}}{\lambda_{-}} A_{k_{\eta}}^{k_{\perp}}(\tau) e^{\mathrm{i} \theta / 2} J_{m+\frac{1}{2}}\left(k_{\perp} \rho\right)
\end{array}\right]=0,
$$

which is equivalent to

$$
\left[\begin{array}{cc}
\lambda & -\mathcal{T}_{-} \\
\mathcal{T}_{+} & \lambda
\end{array}\right]\left[\begin{array}{c}
A_{k_{\eta}}^{k_{\perp}}(\tau) \\
C_{k_{\eta}}^{k_{\perp}}(\tau)
\end{array}\right]=0
$$


The solutions of the above equations are Bessel functions of complex order (and real argument) 21]. So, similarly to Eq. 3.15), the Dirac field in the Cartesian tetrad representation becomes

$$
\begin{aligned}
\psi_{m, k_{\eta}}^{k_{\perp} ; s}=\mathrm{i} \gamma^{1} \gamma^{2} \Phi_{m, k_{\eta}}^{k_{\perp} ; s}=N^{k_{\perp} ; s} e^{\mathrm{i}\left(m \theta+k_{\eta} \eta\right)} \\
\times\left[\begin{array}{l}
-e^{-\mathrm{i}(\theta-\mathrm{i} \eta) / 2} J_{\mathrm{i} k_{\eta}-\frac{1}{2}}(\lambda \tau) J_{m-\frac{1}{2}}\left(k_{\perp} \rho\right) \\
\frac{k_{\perp}}{\lambda_{-}} e^{\mathrm{i}(\theta-\mathrm{i} \eta) / 2} J_{\mathrm{i} k_{\eta}+\frac{1}{2}}(\lambda \tau) J_{m+\frac{1}{2}}\left(k_{\perp} \rho\right) \\
-e^{-\mathrm{i}(\theta+\mathrm{i} \eta) / 2} J_{\mathrm{i} k_{\eta}+\frac{1}{2}}(\lambda \tau) J_{m-\frac{1}{2}}\left(k_{\perp} \rho\right) \\
\frac{k_{\perp}}{\lambda_{-}} e^{\mathrm{i}(\theta+\mathrm{i} \eta) / 2} J_{\mathrm{i} k_{\eta}-\frac{1}{2}}(\lambda \tau) J_{m+\frac{1}{2}}\left(k_{\perp} \rho\right)
\end{array}\right] .
\end{aligned}
$$

Requiring the usual positive/negative-energy boundary conditions 20], we obtain

$$
\begin{aligned}
\psi_{m, k_{\eta}}^{k_{\perp} ; s(+)} & =N_{(+)}^{k_{\perp} ; s} e^{\mathrm{i}\left(m \theta+k_{\eta} \eta\right)} \\
\times & {\left[\begin{array}{l}
-e^{-\mathrm{i}(\theta-\mathrm{i} \eta) / 2} H_{\mathrm{i} k_{\eta}-\frac{1}{2}}^{(2)}(\lambda \tau) J_{m-\frac{1}{2}}\left(k_{\perp} \rho\right) \\
\frac{k_{\perp}}{\lambda_{-}} e^{\mathrm{i}(\theta-\mathrm{i} \eta) / 2} H_{\mathrm{i} k_{\eta}+\frac{1}{2}}^{(2)}(\lambda \tau) J_{m+\frac{1}{2}}\left(k_{\perp} \rho\right) \\
-e^{-\mathrm{i}(\theta+\mathrm{i} \eta) / 2} H_{\mathrm{i} k_{\eta}+\frac{1}{2}}^{(2)}(\lambda \tau) J_{m-\frac{1}{2}}\left(k_{\perp} \rho\right) \\
\frac{k_{\perp}}{\lambda_{-}} e^{\mathrm{i}(\theta+\mathrm{i} \eta) / 2} H_{\mathrm{i} k_{\eta}-\frac{1}{2}}^{(2)}(\lambda \tau) J_{m+\frac{1}{2}}\left(k_{\perp} \rho\right)
\end{array}\right], }
\end{aligned}
$$

and

$$
\begin{aligned}
\psi_{m, k_{\eta}}^{k_{\perp} ; s(-)} & =N_{(-)}^{k_{\perp} ; s} e^{\mathrm{i}\left(m \theta+k_{\eta} \eta\right)} \\
\times & {\left[\begin{array}{l}
-e^{-\mathrm{i}(\theta-\mathrm{i} \eta) / 2} H_{\mathrm{i} k_{\eta}-\frac{1}{2}}^{(1)}(\lambda \tau) J_{m-\frac{1}{2}}\left(k_{\perp} \rho\right) \\
\frac{k_{\perp}}{\lambda_{-}} e^{\mathrm{i}(\theta-\mathrm{i} \eta) / 2} H_{\mathrm{i} k_{\eta}+\frac{1}{2}}^{(1)}(\lambda \tau) J_{m+\frac{1}{2}}\left(k_{\perp} \rho\right) \\
-e^{-\mathrm{i}(\theta+\mathrm{i} \eta) / 2} H_{\mathrm{i} k_{\eta}+\frac{1}{2}}^{(1)}(\lambda \tau) J_{m-\frac{1}{2}}\left(k_{\perp} \rho\right) \\
\frac{k_{\perp}}{\lambda_{-}} e^{\mathrm{i}(\theta+\mathrm{i} \eta) / 2} H_{\mathrm{i} k_{\eta}-\frac{1}{2}}^{(1)}(\lambda \tau) J_{m+\frac{1}{2}}\left(k_{\perp} \rho\right)
\end{array}\right] . }
\end{aligned}
$$

The canonical quantization of the Dirac field is obtained as

$$
\begin{aligned}
\hat{\psi}(\tau, \rho, \theta, \eta)= & \int k_{\perp} \mathrm{d} k_{\perp} \int \mathrm{d} k_{\eta} \sum_{m=-\infty}^{\infty} \sum_{s} \\
& \times\left[\psi_{m, k_{\eta}}^{k_{\perp} ; s(+)} a_{m, k_{\eta}}^{k_{\perp} ; s}+\psi_{m, k_{\eta}}^{k_{\perp} ; s(-)} b_{m, k_{\eta}}^{k_{\perp} ; s^{\dagger}}\right] .
\end{aligned}
$$

where $\psi_{m, k_{\eta}}^{k_{\perp} ; s( \pm)}$ correspond to the positive and negative energy solutions, respectively. The normalization constants $N_{( \pm)}^{k_{\perp} ; s}$ are obtained by requiring the fields $\psi_{m, k_{\eta}}^{k_{\perp} ; s( \pm)}$ to satisfy the orthonormality relations

$$
\begin{aligned}
\left(\bar{\psi}_{m, k_{\eta}}^{k_{\perp} ; s( \pm)}, \psi_{m^{\prime}, k_{\eta}^{\prime}}^{k^{\prime} ; s^{\prime}( \pm)}\right) & =\frac{\delta\left(k_{\perp}^{\prime}-k_{\perp}\right)}{k_{\perp}} \delta\left(k_{\eta}^{\prime}-k_{\eta}\right) \delta_{m^{\prime} m} \delta_{s^{\prime} s}, \\
\left(\bar{\psi}_{m, k_{\eta}}^{k_{\perp} ;(+)}, \psi_{m^{\prime}, k_{\eta}^{\prime}}^{k_{\perp}^{\prime} ; s^{\prime}(-)}\right) & =0 .
\end{aligned}
$$

where we introduce the inner product

$$
\left(\bar{\psi}_{m, k_{\eta}}^{k_{\perp} ; s}, \psi_{m^{\prime}, k_{\eta}^{\prime}}^{k_{\perp}^{\prime} ; s^{\prime}}\right)=-\tau \int \rho \mathrm{d} \rho \int \mathrm{d} \theta \int \mathrm{d} z \bar{\psi}_{m, k_{\eta}}^{k_{\perp} ; s} \tilde{\gamma}^{0} \psi_{m^{\prime}, k_{\eta}^{\prime}}^{k_{\prime}^{\prime} ; s^{\prime}} .
$$

We find the normalization constants

$$
N_{( \pm)}^{k_{\perp} ; s}=\frac{1}{2 \pi} \sqrt{\frac{M(\lambda-M)}{16 \lambda}} e^{ \pm \pi k_{\eta} / 2} .
$$

\section{B. Diagonal tetrad representation}

In the diagonal tetrad representation the Dirac equation takes the form

$$
\begin{aligned}
{\left[\gamma^{0}\left(\partial_{\tau}+\frac{1}{2 \tau}\right)\right.} & +\gamma^{1}\left(\partial_{\rho}+\frac{1}{2 \rho}\right) \\
& \left.+\gamma^{2} \frac{1}{\rho} \partial_{\theta}+\gamma^{3} \frac{1}{\tau} \partial_{\eta}+M\right] \psi_{[\mathrm{d}]}=0
\end{aligned}
$$

and has a solution of the form

$$
\psi_{[d]}=\int k_{\perp} \mathrm{d} k_{\perp} \int \mathrm{d} k_{\eta} \sum_{m=-\infty}^{\infty} \sum_{s} \psi_{m, k_{\eta}[\mathrm{d}]}^{k_{\perp} ; s},
$$

with

$$
\begin{aligned}
\psi_{m, k_{\eta}[\mathrm{d}]}^{k_{\perp} ; s}=\mathrm{i} & \gamma^{1} \gamma^{2} \Phi_{m, k_{\eta}}^{k_{\perp} ; s}=N^{k_{\perp} ; s} e^{\mathrm{i}\left(m \theta+k_{\eta} \eta\right)} \\
\times & {\left[\begin{array}{r}
-J_{\mathrm{i} k_{\eta}-\frac{1}{2}}(\lambda \tau) J_{m-\frac{1}{2}}\left(k_{\perp} \rho\right) \\
\frac{k_{\perp}}{\lambda_{-}} J_{\mathrm{i} k_{\eta}+\frac{1}{2}}(\lambda \tau) J_{m+\frac{1}{2}}\left(k_{\perp} \rho\right) \\
-J_{\mathrm{i} k_{\eta}+\frac{1}{2}}(\lambda \tau) J_{m-\frac{1}{2}}\left(k_{\perp} \rho\right) \\
\frac{k_{\perp}}{\lambda_{-}} J_{\mathrm{i} k_{\eta}-\frac{1}{2}}(\lambda \tau) J_{m+\frac{1}{2}}\left(k_{\perp} \rho\right)
\end{array}\right] . }
\end{aligned}
$$

Then, the positive- and negative-energy solutions correspond to

$$
\begin{aligned}
\psi_{m, k_{\eta}[\mathrm{d}]}^{k_{\perp} ; s(+)}= & N_{(+)}^{k_{\perp} ; s} e^{\mathrm{i}\left(m \theta+k_{\eta} \eta\right)} \\
& \times\left[\begin{array}{c}
-H_{\mathrm{i} k_{\eta}-\frac{1}{2}}^{(2)}(\lambda \tau) J_{m-\frac{1}{2}}\left(k_{\perp} \rho\right) \\
\frac{k_{\perp}}{\lambda_{-}} H_{\mathrm{i} k_{\eta}+\frac{1}{2}}^{(2)}(\lambda \tau) J_{m+\frac{1}{2}}\left(k_{\perp} \rho\right) \\
-H_{\mathrm{i} k_{\eta}+\frac{1}{2}}^{(2)}(\lambda \tau) J_{m-\frac{1}{2}}\left(k_{\perp} \rho\right) \\
\frac{k_{\perp}}{\lambda_{-}} H_{\mathrm{i} k_{\eta}-\frac{1}{2}}^{(2)}(\lambda \tau) J_{m+\frac{1}{2}}\left(k_{\perp} \rho\right)
\end{array}\right],
\end{aligned}
$$

and

$$
\begin{aligned}
\psi_{m, k_{\eta}[\mathrm{d}]}^{k_{\perp} ; s(-)}= & N_{(-)}^{k_{\perp} ; s} e^{\mathrm{i}\left(m \theta+k_{\eta} \eta\right)} \\
& \times\left[\begin{array}{c}
-H_{\mathrm{i} k_{\eta}-\frac{1}{2}}^{(1)}(\lambda \tau) J_{m-\frac{1}{2}}\left(k_{\perp} \rho\right) \\
\frac{k_{\perp}}{\lambda_{-}} H_{\mathrm{i} k_{\eta}+\frac{1}{2}}^{(1)}(\lambda \tau) J_{m+\frac{1}{2}}\left(k_{\perp} \rho\right) \\
-H_{\mathrm{i} k_{\eta}+\frac{1}{2}}^{(1)}(\lambda \tau) J_{m-\frac{1}{2}}\left(k_{\perp} \rho\right) \\
\frac{k_{\perp}}{\lambda_{-}} H_{\mathrm{i} k_{\eta}-\frac{1}{2}}^{(1)}(\lambda \tau) J_{m+\frac{1}{2}}\left(k_{\perp} \rho\right)
\end{array}\right],
\end{aligned}
$$

where $N_{( \pm)}^{k_{\perp} ; s}$ is given by Eq. (5.22). The Dirac fields in the Cartesian and diagonal tetrad representations are related via the similarity transformation (5.9). We note that $S_{\rho}$ is given by (4.61), and $S_{\tau}$ is defined as

$$
S_{\tau}=\left[\begin{array}{cccc}
e^{-\eta / 2} & 0 & 0 & 0 \\
0 & e^{\eta / 2} & 0 & 0 \\
0 & 0 & e^{\eta / 2} & 0 \\
0 & 0 & 0 & e^{-\eta / 2}
\end{array}\right]
$$

We can easily verify that indeed we have

$$
\psi_{m, k_{\eta}}^{k_{\perp} ; s}=S_{\tau} S_{\rho} \psi_{m, k_{\eta}[d]}^{k_{\perp} ; s} .
$$




\section{CONCLUSIONS}

In this paper we have shown step by step how to obtain the canonical quantization in cylindrical geometry as well as in the $1+1$ dimensional fluid rapidity and proper time coordinates. Assuming that we are interested in a quantum back-reaction problem with a background chromoelectric field that is a function only of $\tau$, then the separation of variables we have made will continue for the problem of interest, and the initial value problem choice that $A\left(\tau_{0}\right)=0$, tells us that the correct mode expansion at $\tau=\tau_{0}$ for the Dirac equation is given by the problem we have solved. Thus the canonical quantization presented here for the Dirac equation, will allow us to determine the initial conditions for the quark state (adiabatic vacuum) so that we will be able to numerically solve for the production of quarks and the degradation of the chromoelectric field in a manner similar to the problem addressed by Cooper et al. in Ref. [6]. By additionally having the correct modes for the transverse degrees of freedom, we can exploit the cylindrical symmetry and reduce the number of momentum modes needed in the $3+1$ dimensional problems to be $N^{2}$ instead of $N^{3}$. These numerical simulations will be soon undertaken in the case of color $\mathrm{SU}(3)$ quarks.

\section{Acknowledgments}

The authors would like to thank A.B. Balantekin and A.J. DeWeerd for making available their unpublished work, and we would also like to thank the Santa Fe Institute for its hospitality during the completion of this work.

\section{APPENDIX A: COMMENTS ON THE SOLUTION OF THE DIRAC EQUATION IN CYLINDRICAL COORDINATES}

First, let us note that the same normalization constant $N_{k_{z}, E}^{k_{\perp}}$ is determined by requiring the conditions [15]:

$$
\begin{gathered}
\sum_{m=-\infty}^{\infty} \Phi_{m, k_{z} ;|E|}^{k_{\perp} ; s \dagger}(\rho, \theta) \Phi_{m, k_{z} ;|E|}^{k_{\perp} ; s^{\prime}}(\rho, \theta)=\delta_{s s^{\prime}}, \\
\sum_{m=-\infty}^{\infty} \Phi_{m, k_{z} ;-|E|}^{k_{\perp} ; s \dagger}(\rho, \theta) \Phi_{m, k_{z} ;-|E|}^{k_{\perp} ; s^{\prime}}(\rho, \theta)=\delta_{s s^{\prime}}
\end{gathered}
$$

The following conditions are enforced by construction

$$
\begin{gathered}
\sum_{m=-\infty}^{\infty} \Phi_{m, k_{z} ;|E|}^{k_{\perp} ; \dagger}(\rho, \theta) \Phi_{m, k_{z} ;-|E|}^{k_{\perp} ; s^{\prime}}(\rho, \theta)=0, \\
\sum_{m=-\infty}^{\infty} \Phi_{m, k_{z} ;-|E|}^{k_{\perp} ; s \dagger}(\rho, \theta) \Phi_{m, k_{z} ;|E|}^{k_{\perp} ; s^{\prime}}(\rho, \theta)=0 .
\end{gathered}
$$

In using the above conditions one makes use of Bessel functions' property (see discussion regarding the Graf generalization of Neumann's formula in Ref. 22] ):

$$
\sum_{m=-\infty}^{+\infty} J_{m+\nu}(x) J_{m}(y) e^{ \pm \mathrm{i} m \theta}=e^{ \pm \mathrm{i} \nu \phi} J_{\nu}(z)
$$

where

$$
\begin{array}{r}
z=\sqrt{x^{2}+y^{2}-2 x y \cos \theta}, \\
x-y \cos \theta=z \cos \phi, \\
y \sin \theta=z \sin \phi .
\end{array}
$$

Secondly, we discuss the boundary conditions in the $(x, y)$ subspace. Provided that we require the solution to be regular at the origin, then the generic form (4.27) reads as follows: for $m \geq 1$, we have

$$
\Phi_{m}^{k_{\perp} ; s}(\rho, \theta)=e^{\mathrm{i} m \theta}\left[\begin{array}{r}
A^{k_{\perp}} e^{-\mathrm{i} \theta / 2} J_{m-\frac{1}{2}}\left(k_{\perp} \rho\right) \\
\frac{k_{\perp}}{\lambda_{-}} C^{k_{\perp}} e^{\mathrm{i} \theta / 2} J_{m+\frac{1}{2}}\left(k_{\perp} \rho\right) \\
C^{k_{\perp}} e^{-\mathrm{i} \theta / 2} J_{m-\frac{1}{2}}\left(k_{\perp} \rho\right) \\
\frac{k_{\perp}}{\lambda_{-}} A^{k_{\perp}} e^{\mathrm{i} \theta / 2} J_{m+\frac{1}{2}}\left(k_{\perp} \rho\right)
\end{array}\right],
$$

whereas for $m \leq-1$ we have

$$
\Phi_{m}^{k_{\perp} ; s}(\rho, \theta)=e^{\mathrm{i} m \theta}\left[\begin{array}{r}
A^{k_{\perp}} e^{-\mathrm{i} \theta / 2} Y_{m-\frac{1}{2}}\left(k_{\perp} \rho\right) \\
\frac{k_{\perp}}{\lambda_{-}} C^{k_{\perp}} e^{\mathrm{i} \theta / 2} Y_{m+\frac{1}{2}}\left(k_{\perp} \rho\right) \\
C^{k_{\perp}} e^{-\mathrm{i} \theta / 2} Y_{m-\frac{1}{2}}\left(k_{\perp} \rho\right) \\
\frac{k_{\perp}}{\lambda_{-}} A^{k_{\perp}} e^{\mathrm{i} \theta / 2} Y_{m+\frac{1}{2}}\left(k_{\perp} \rho\right)
\end{array}\right],
$$

where $Y_{\nu}(x)$ is the Neumann function. For integer $m$ we have the property 22]

$$
\begin{aligned}
& Y_{m-\frac{1}{2}}(x)=(-)^{m} J_{-m+\frac{1}{2}}(x), \\
& Y_{m+\frac{1}{2}}(x)=(-)^{m+1} J_{-m-\frac{1}{2}}(x) .
\end{aligned}
$$

Finally, when $m=0$ the solution (4.27) becomes

$$
\Phi_{m}^{k_{\perp} ; s}(\rho, \theta)=\left[\begin{array}{c}
A^{k_{\perp}} e^{-\mathrm{i} \theta / 2} J_{-\frac{1}{2}}\left(k_{\perp} \rho\right) \\
\frac{k_{\perp}}{\lambda_{-}} C^{k_{\perp}} e^{\mathrm{i} \theta / 2} Y_{\frac{1}{2}}\left(k_{\perp} \rho\right) \\
C^{k_{\perp}} e^{-\mathrm{i} \theta / 2} J_{-\frac{1}{2}}\left(k_{\perp} \rho\right) \\
\frac{k_{\perp}}{\lambda_{-}} A^{k_{\perp}} e^{\mathrm{i} \theta / 2} Y_{\frac{1}{2}}\left(k_{\perp} \rho\right)
\end{array}\right]
$$

It is interesting to note that the discussion of the boundary conditions in the $(x, y)$ subspace simplifies dramatically if we choose Bessel functions of integer rather than half-integer order in (4.27). In other words, in the $(x, y)$ subspace we can replace the form (4.27) which involves Bessel functions of half-integer order, and use instead the form

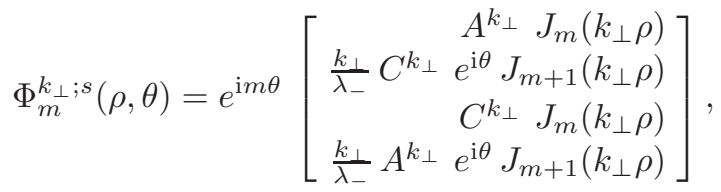


which has the correct boundary conditions irrespective of the value of $m$. The fact that Eq. (4.12) allows for a solution of the form (A14) is unlike the case of $(t, z)$ subspace where the form of the solution (3.15) is mandatory 24 in order to satisfy the orthonormality conditions (3.19).
[1] J. D. Bjorken, Phys. Rev. D 27, 140 (1983)

[2] L. D. Landau, Izv. Akad. Nauk SSSR (Ser. Fiz.) 17, 51 (1953).

[3] Fred Cooper, Div. of Particles and Fields Meeting of American Physical Society, Williamsburg, Va, Sep 5-7, 1974, pp. 499-531; F. Cooper, G. Frye, and E. Schonberg, Phys. Rev. D 11, 192 (1975).

[4] R. C. Hwa, Phys. Rev. D 10, 2260 (1974)

[5] J. Schwinger, Phys. Rev. 82, 664 (1951).

[6] F. Cooper, J.M. Eisenberg, Y. Kluger, E. Mottola, and B. Svetitsky, Phys. Rev. D 48, 190 (1993).

[7] G.C. Nayak and P. van Nieuwenhuizen, Phys. Rev. D 71, 125001 (2005); G.C. Nayak, Phys. Rev. D 72, 125010 (2005); F. Cooper and G.C. Nayak, Phys. Rev. D 73, 065005 (2006).

[8] D. Brill and J. Wheeler, Rev. Mod. Phys. 29, 465 (1957)

[9] L. Parker, Phys. Rev. D 3, 346 (1971); S.A. Fulling, L. Parker, and B.L. Hu, ibid., 10, 3905 (1974).

[10] G.V. Shishkin and V.M. Villalba, J. Math. Phys. 30, 2132 (1989); V.M. Villalba, J. Math. Phys. 31, 2702 (1990); J. Phys. A: Math. Gen. 24, 3781 (1991).

[11] G.V. Shishkin and V.M. Villalba, J. Math. Phys. 34, 5037 (1993); V.M. Villalba, J. Math. Phys. 30, 2373 (1989); Il Nuovo Cimento 112B, 109 (1997); V.M. Villalba and E.I. Catalá, J. Math. Phys. 31, 715 (1990); V.M. Villalba and E.I. Catalá, J. Math. Phys. 43, 4909 (2002).

[12] C.M. Sommerfield, Ann. Phys. (N.Y.) 84, 285 (1974)

[13] A. di Sessa, J. Math. Phys., 15, 1892 (1974); Phys. Rev. D 9, 2926 (1974).

[14] D. Grome, H. Rothe, and B. Stech, Nucl. Phys. B 75, 313 (1974)

[15] A.B. Balantekin and A.J. DeWeerd, "Second quantization of the Dirac equation in cylindrical coordinates," (unpublished) (1995).

[16] H. Weyl, Z. Phys. (Germany) 56, 330 (1929).

[17] J.M. Jauch and F. Rohrlich, The Theory of Photons and Electrons, p. 425, (Springer, New York, 1976).

[18] N.D. Birrell and P.C.W. Davies, Quantum Fields in
Curved Space, p. 84, (Cambridge University Press, Cambrisge, 1982).

[19] S. Weinberg, Gravitation and Cosmology: Principles and Applications of the General Theory of Relativity, (John Wiley \& Sons, New York, 1972).

[20] Positive- and negative-energy solutions, $\psi_{k}^{( \pm)}(x)$, are defined such that $\psi_{k}^{( \pm)}(x)$ vanish in the limit $t \rightarrow \mp \mathrm{i} \infty$, respectively [13]. The Hankel functions of real argument $H_{\nu}^{(1,2)}(x)$ have the required properties. Hence, $\psi_{k}^{(+)}(x) \propto$ $H_{\nu}^{(2)}(x)$ whereas $\psi_{k}^{(-)}(x) \propto H_{\nu}^{(1)}(x)$.

[21] If $\operatorname{Re} z>0$, then we can use the integral representation of the Bessel function $J_{\nu}(z)=\frac{1}{2 \pi} \int_{\pi-\infty \mathrm{i}}^{\pi+\infty \mathrm{i}} \exp [-\mathrm{i} z \sin \theta+$ $\mathrm{i} \nu \theta] \mathrm{d} \theta$, where the path of integration is taken around the semi-infinite strip $y \geq 0,-\pi \leq x \leq \pi-$ see e.g. I.S. Gradshteyn and I.M. Ryzhik, Table of integrals, series, and products, sixth edition, p. 903 (Academic Press, San Diego, 2000).

[22] G.N. Watson, A Treatise on the Theory of Bessel Functions, second edition, (Cambridge University Press, Cambrisge, 1966).

[23] Y. Kluger, J.M. Eisenberg, B. Svetitsky, F. Cooper, and E. Mottola, Phys. Rev. D 45, 4659 (1992).

[24] The natural choice for the amplitude $\psi_{k_{\eta}}$ has $J_{\mathrm{i} k_{\eta}}$ and $-e^{\eta} J_{\mathrm{i} k_{\eta}+1}$ as the upper and lower components, respectively. However, this choice results in a modified form of the orthonormality relations (3.19), which involves $\delta\left(k_{\eta}^{\prime}-k_{\eta}-\mathrm{i}\right)$. This suggests that the upper and lower components in $\psi$ must be "symmetrized" with respect to $\eta$.

[25] Since the gamma matrices anticommute, $\left\{\gamma^{\mu}, \gamma^{\nu}\right\}=0$ for $\mu \neq \nu$, it follows that a factor i $\gamma^{1} \gamma^{2}$ is necessary in order to make the operators $\mathcal{K}_{1}$ and $\mathcal{K}_{1}$ commute. For this to happen, the term containing the mass $M$ must be included in $\mathcal{K}_{2}$, and $\mathcal{K}_{2}$ gives the form of the Dirac spinor. If instead we use the factor i $\gamma^{0} \gamma^{3}$, then the mass must be in $\mathcal{K}_{1}$, and the form of the Dirac spinor is given by solving the eigenvalue problem for $\mathcal{K}_{1}$. 